\title{
Millimeter-Wave Microstrip Antenna Array Design and an Adaptive Algorithm for Future 5G Wireless Communication Systems
}

\author{
Cheng-Nan Hu, ${ }^{1}$ Dau-Chyrh Chang, ${ }^{1}$ Chung-Hang Yu, ${ }^{2}$ \\ Tsai-Wen Hsaio, ${ }^{2}$ and Der-Phone Lin ${ }^{2}$ \\ ${ }^{1}$ Communication Engineering Department, OIT, New Taipei City 22061, Taiwan \\ ${ }^{2}$ National Chung-Shan Institute of Science and Technology, P.O. Box 90008, Longtan, Taoyuan City, Taiwan \\ Correspondence should be addressed to Cheng-Nan Hu; fo011@mail.oit.edu.tw
}

Received 9 September 2016; Accepted 14 November 2016

Academic Editor: Atiqur Rahman

Copyright (C) 2016 Cheng-Nan Hu et al. This is an open access article distributed under the Creative Commons Attribution License, which permits unrestricted use, distribution, and reproduction in any medium, provided the original work is properly cited.

This paper presents a high gain millimeter-wave $(\mathrm{mmW})$ low-temperature cofired ceramic (LTCC) microstrip antenna array with a compact, simple, and low-profile structure. Incorporating minimum mean square error (MMSE) adaptive algorithms with the proposed 64-element microstrip antenna array, the numerical investigation reveals substantial improvements in interference reduction. A prototype is presented with a simple design for mass production. As an experiment, HFSS was used to simulate an antenna with a width of $1 \mathrm{~mm}$ and a length of $1.23 \mathrm{~mm}$, resonating at $38 \mathrm{GHz}$. Two identical $\mathrm{mmW}$ LTCC microstrip antenna arrays were built for measurement, and the center element was excited. The results demonstrated a return loss better than $15 \mathrm{~dB}$ and a peak gain higher than $6.5 \mathrm{dBi}$ at frequencies of interest, which verified the feasibility of the design concept.

\section{Introduction}

The growing demands on data traffic are propelling the evolution of wireless standards. Soon after the first forzon release in December 2008, commercial development of the 3rdGeneration Partnership Project (3GPP) Long-Term Evolution (LTE) system began. To increase capacity, LTE-Advanced was launched in March 2011, rendering LTE formally compliant with the International Telecommunication Union's definition of the fourth generation of wireless technology, termed IMT-Advanced [1, 2]. However, new traffic types and data services are emerging, notably machine-to-machine communications to support applications such as smart grid, smart homes and cities, and e-health. With the development of new applications, as many as 50 billion devices may be connected by 2020 according to one estimate [3]. The growing annual traffic demands (conservative estimates range from $40 \%$ to $70 \%$ [4-6]) imply that cellular networks may need to deliver as much as 1000 times their current capacity within the next decade. The challenging task of meeting this demand impels the development of future wireless networks, called beyond fourth-generation (4G) and fifth-generation (5G) cellular systems, offering peak throughputs of multigigabits per second $(\mathrm{Gb} / \mathrm{s})$ and cell edge rates in tens of megabits per second $(\mathrm{Mb} / \mathrm{s})[7]$.

In addition to offering greater performance than LTEAdvanced, future networks must be much more energy efficient than current networks for 5G technology to be sustainable in the long term. Millimeter-wave $(\mathrm{mmW})$ beamforming technology is a key enabling factor for the data capacity improvement that is essential for $5 \mathrm{G}$ networks to be commercially successful. This technology employs a massive multi-input multioutput (MIMO) system operating between 30 and $300 \mathrm{GHz}$, where the available bandwidths are much wider than current cellular networks [8-11]. The new spectrum released by the Federal Communications Commission features bandwidth up to $11 \mathrm{GHz}$, including $3.8 \mathrm{GHz}$ of licensed spectrum from 27.5 to $28.35 \mathrm{GHz}$ and 37 to $40 \mathrm{GHz}$ and $7 \mathrm{GHz}$ of unlicensed spectrum from 64 to $71 \mathrm{GHz}$. The available spectrum of the $\mathrm{mmW}$ band can achieve data rates 


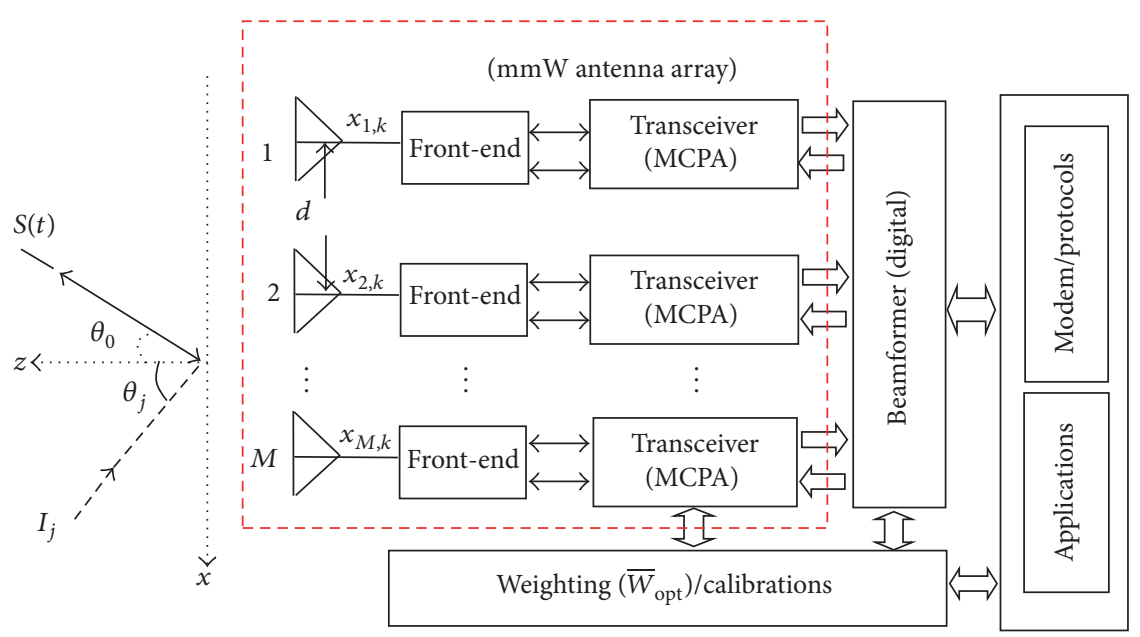

FIGURE 1: The functional block diagram of an adaptive antenna array for massive MIMO systems.

200 times greater than those realized by current cellular systems under $3 \mathrm{GHz}[8,10]$. Furthermore, the relatively short wavelength of an $\mathrm{mmW}$ signal, which incorporates leading radiofrequency integrated circuit technologies, renders the implementation of an antenna with numerous $(\geq 32)$ elements with small dimension possible. Consequently, future wireless networks can be equipped with massive MIMO systems to provide an extremely high gain, electrically steerable array, and adaptive beamforming arrays. Furthermore, these systems can be built in the base station, on the terminal device, or within a chip $[8,12]$.

Despite the benefits of using the mmW band, numerous substantial challenges must be addressed before the wireless systems in these bands can be realized, especially for the array antenna design.

(1) In the mmW region, designing electromagnetic circuit elements is difficult because of the sensitivity in fabrication requirements and the high insertion loss in wave propagation. Slight misalignments of parameters on the order of $0.1 \mathrm{~mm}$ can change the characteristics of the circuit element and cause unexpected consequences.

(2) Greater antenna gain for the same physical antenna size is attained because of the smaller wavelength of the $\mathrm{mmW}$ signal. However, the reliance on highly directional transmissions necessitates design changes to current cellular systems [13].

(3) Fabricating structures and fixing connectors when transitioning to the other transmission lines require care. The fringing fields over the transition region on the panel launch transmission line feed have capacitive or inductive input impedance resulting in distortion and poor matching performance.

This paper reports on the recent progress in designing a prototype $\mathrm{mmW}$ antenna array for massive MIMO applications; the array involves a 64-element microstrip antenna array operating at $38 \mathrm{GHz}$. In Section 2.1, the system configuration of the proposed mmW microstrip antenna array is discussed in detail. This section also outlines the minimum mean square error $[14,15]$ and least mean squares (LMS) [14] adaptive algorithms, which show great improvement in signal-to-noise ratio (SNR) by placing nulls in interference directions. Section 2.2 presents the design of a prototype mmW antenna array using a microstrip patch antenna printed on a low-temperature cofired ceramic (LTCC) substrate. Section 3 describes the experimental study of the mmW microstrip antenna arrays, namely, 64-element microstrip patch antennas and a reference antenna for self-calibration [16]. Concluding remarks are provided in Section 4.

\section{Analysis and Design}

2.1. System Configuration. The functional block diagram of an $M$-element massive MIMO system for future networks is depicted in Figure 1. During transmission, the optimal weighting vector $\left(\mathbf{W}_{\text {opt }}\right)$ is computed to generate high gain radiation fields along the signal directions $\left(\theta_{0}, \phi_{0}\right)$ and synthesize nulls on other unlined user equipment (UE) directions $\left(\theta_{j}, \phi_{j}\right)$; this minimizes channel interference. Therefore, when the SNR increases, a high-quality signal is achieved in the radio line between BS and UE for high-throughput performance. Similarly, the incoming signal and interference in the directions received by the array are amplified, downconverted, and transformed to the baseband signal from analog to digital format. While the radiation pattern is synthesized with the high gain in the signal direction and nulls in the interference directions, a closed-loop adaptive processor modifies the received signals in a fading environment by computing the optimal weighting vector $\left(\mathbf{W}_{\text {opt }}\right)$. An equally spaced $d$-space $M$-element antenna array is subjected to this process.

Figure 2 illustrates the design concept of the proposed mmW active antenna array for massive MIMO systems, indicating that it comprises the following components: (1) 64element microstrip patch antennas; (2) 16 front-end modules; 


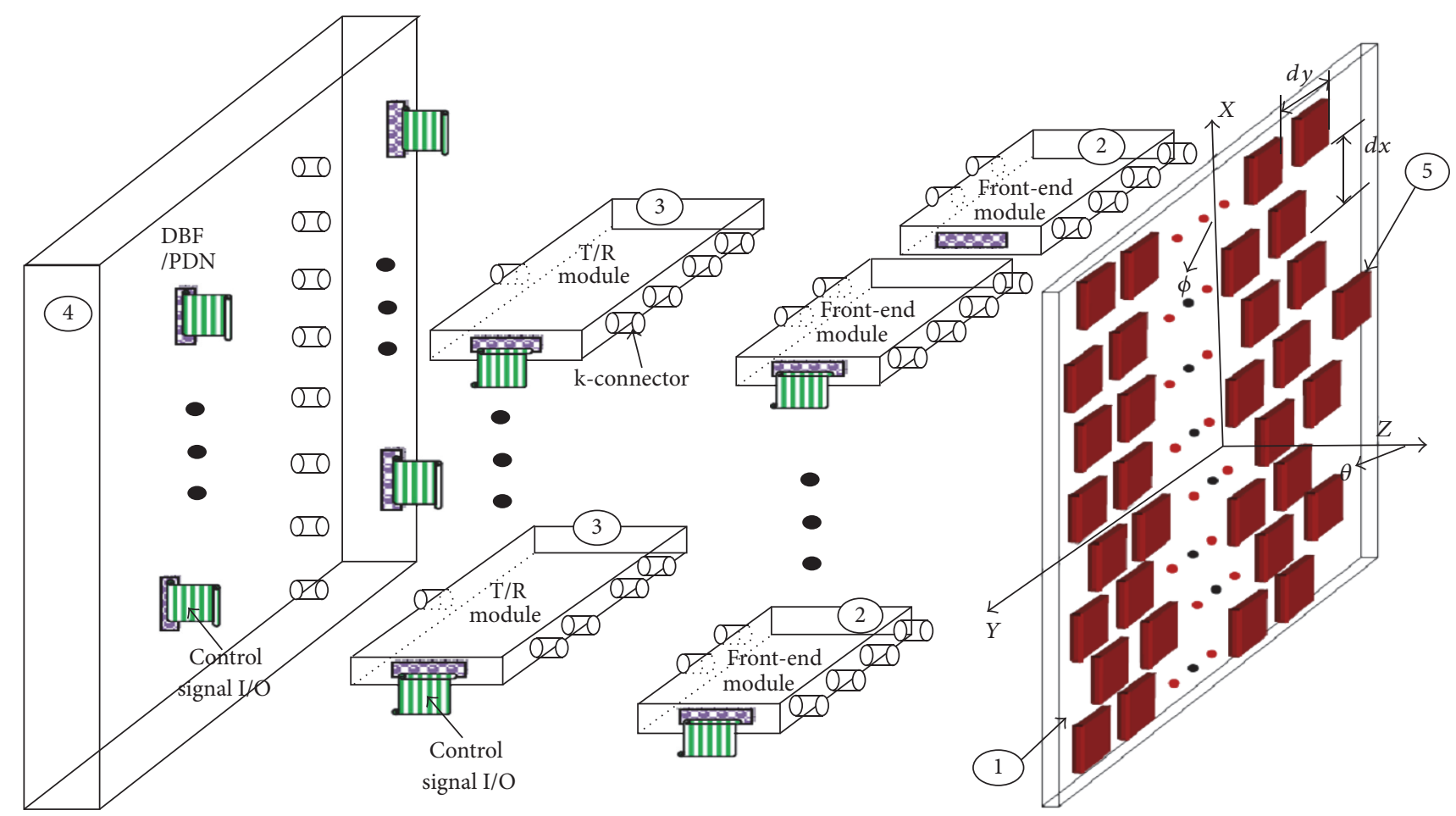

(a)

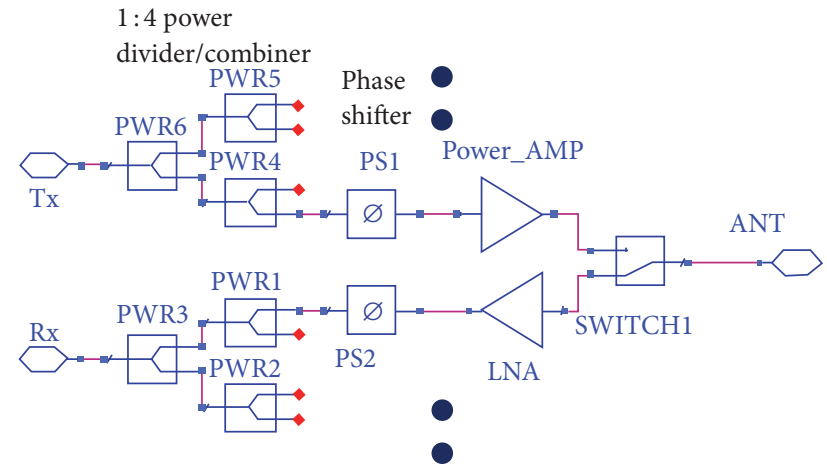

(b)

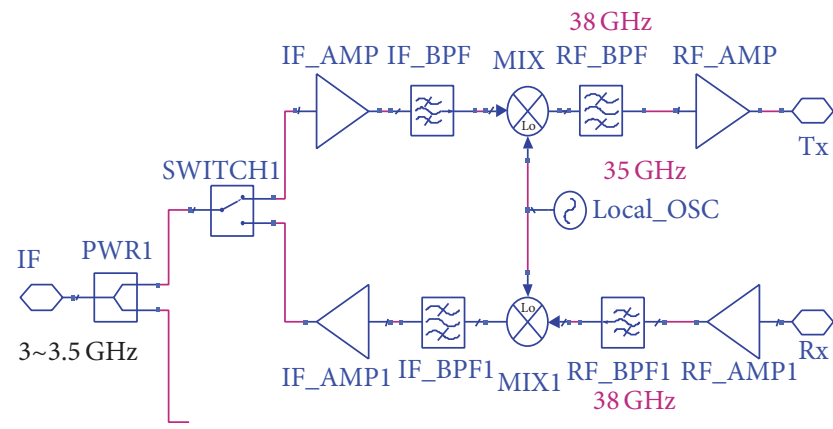

(c)

FIGURE 2: (a) Design concept of the mmW active antenna array for massive MIMO systems, comprising (1) 64-element microstrip patch antennas, (2) 16 front-end modules, (3) eight T-R modules, (4) an eight-channel digital beamforming network and power distribution network, and (5) a reference antenna integrated with a T-R module. (b) Schematic of the front-end module on one channel. (c) Schematic of the T-R module on one channel.

(3) eight transmitter-receiver (T-R) modules; (4) a power distribution network; and (5) a reference antenna integrated with a T-R module, which is applied to the system selfcalibration using the phase-match method [16].

The antenna array comprises a set of polarized elements in the position vector $\left(D=\left[\mathbf{d}_{1}, \mathbf{d}_{2}, \ldots, \mathbf{d}_{\mathbf{m}}, \ldots, \mathbf{d}_{M}\right]\right)$, where $\mathbf{d}_{\mathbf{m}}$ denotes the location of the $m$ th antenna element. For plane waves that propagate in a locally homogeneous medium, the wave $\mathbf{K}$ is defined as

$$
\mathbf{K}=\frac{2 \pi}{\lambda}[\sin \theta \cos \phi \sin \theta \sin \phi \cos \theta]^{T},
$$

where $\lambda$ represents the wavelength at frequency $f ; \theta$ and $\phi$ are the azimuthal angle and angle of elevation of the far-field source, respectively; and $T$ denotes the matrix transpose.

When the time phase factor $e^{j 2 \pi f t}$ is omitted, the far-field pattern of the simultaneously excited adaptive antenna array becomes

$$
f(\theta, \phi)=\mathbf{W}^{H} \cdot e^{-j \mathbf{K}^{T} \cdot \mathbf{D}} \xi(\theta, \phi)=\mathbf{W}^{H} \cdot \mathbf{E}(\theta, \phi),
$$

where

$$
\begin{aligned}
\mathbf{W} & =\left[w_{1}, w_{2}, \ldots, w_{M}\right]^{T}, \\
\mathbf{E}(\theta, \phi) & =\mathbf{e}^{-\mathbf{j} \mathbf{K}^{\mathrm{T}} \cdot \mathbf{D}} \xi(\theta, \phi) .
\end{aligned}
$$


The polarized element pattern function for an individual element is denoted by $\xi(\theta, \phi)$, and $w_{i}\left(w_{i}=c_{i} e^{j \psi_{i}}\right)$ represents the complex weighting coefficient with an amplitude of $c_{i}$ and phase of $\Psi_{i}$ at the $i$ th element of the weight vector $\mathbf{W}$, which is generated by the adaptive algorithm. The Hermitian transpose, which combines transposition and conjugation, is denoted by $\mathbf{H}$, and the radiation pattern of the array is denoted by $f(\theta, \phi)$. For a given radial vector (defined by angles $\phi$ and $\theta), f(\theta, \phi)$ yields the magnitude of the array response in the $\widehat{\phi}$ and $\widehat{\theta}$ directions.

The system performance enhancement of the massive MIMO system was assessed using adaptive algorithms in a noisy environment. The received signal of a single antenna in an additive white Gaussian noise channel is represented by $x(t)=S(t)+n(t)$, where $S(t)$ denotes the received signal in the direction $\left(\theta_{0}, \phi_{0}\right)$ and $n(t)$ denotes the noise. The adaptive beamformer with the selected algorithm is then derived using the conventional phased array approach at radiofrequency (RF) or intermediate frequency (IF) $[14,15]$. The signals collected from each of the antenna elements are processed and summed in the analog devices and downconverted to the baseband. The weighting process in the RF and IF analog domains is relatively inflexible and can become complex as the number of space-division multiple-access channels increases. The digital-domain, or digital beamforming (DBF), approach has numerous advantages $[17,18]$ but is also disadvantaged by its unavailability, high cost, increased signal bandwidth, and the dynamic range of characteristics of analog-to-digital and digital-to-analog converters.

In this paper, the mean squared error (MSE) is minimized by using the LMS algorithm $[14,19,20]$ as an example of selected adaptive DBF. In discrete time, at time index $k$ and period $T_{s}, t=k T_{s}$, the input signal vector of an equally spaced $M$-element array (Figure 1) is

$$
\mathbf{X}_{k}=\left[x_{1, k}, x_{2, k}, \ldots, x_{m, k}, \ldots, x_{M, k}\right]
$$

where $x_{m, k}$ is the transmitted narrowband signal in a complex envelope and $m$ is the number of antenna elements at $k$. Moreover, $\mathbf{W}_{k}$ is the weight vector at $k$, which can be applied in the analog portion of the receiver using phase shifters and attenuators, or in the digital domain after digitizing and filtering the signal. For narrowband arrays, the output is the linear combination of the sampled inputs that are multiplied with complex weights:

$$
y_{k}=\mathbf{X}_{k}^{H} \cdot \mathbf{W}_{k} \text {. }
$$

The optimal solution that minimizes MSE leads to the Wiener-Hopf solution [14] and is given by

$$
\mathbf{W}_{k}=\mathbf{R}_{x x}{ }^{-1} \cdot \mathbf{r}_{x d}
$$

where $\mathbf{R}_{x x}$ denotes the input correlation or covariance matrix and $\mathbf{r}_{x d}$ is the cross-correlation vector between the received and training (or desired) signals.
For stationary input data, the process requires only the time to yield adequate estimates of $\mathbf{R}_{x x}$ and $\mathbf{r}_{x d} \cdot \mathbf{R}_{x x}{ }^{-1}$ is then computed and the optimal weight vector is determined. However, because matrix inversion is nontrivial, particularly when conducted using a digital signal processor, iterative techniques easily yield the optimal solution in a limited time. The updated equation of these iterative approaches is generally expressed by the LMS algorithm [18] given by

$$
\mathbf{W}_{\mathbf{k}+1}=\mathbf{W}_{\mathbf{k}}-\Delta\left(\mathbf{G}_{\mathbf{k}}\right)
$$

where $\Delta$ represents the step-size value and is allowed to change at every iteration and $\mathbf{G}_{\mathbf{k}}$ denotes the gradient or derivative of the MSE with respect to the weight vector $\left(\mathbf{W}_{k}\right)$ :

$$
G_{k}=-2 r_{x d}+2 R_{x x} W_{k} .
$$

Stochastic gradient algorithms attempt to determine the optimal solution by continually stepping in the negative direction of the gradient. Thus, the weight vector is adjusted toward the minimum optimal MSE surface. Therefore, the adaptive weighting vector can be computed with the DBF unit (Figure 1) by using (7); the adaptive weighting vector is then fed into transceivers to adjust the amplitude and phase of each patch antenna through the automatic gain controller and phase shifter. Consequently, the far-field pattern of the simultaneously excited adaptive antenna array can be achieved and the SNR can be maximized to optimize the system performance under fading environments.

Figure 3(a) indicates that the computed element gain of a single patch antenna of $6.86 \mathrm{dBi}$, which is achieved using HFSS and the theoretical directivity gain improvement for a massive MIMO system with a 64-element array antenna and element spacing of $d x=5.5 \mathrm{~mm}$ and $d y=8.5 \mathrm{~mm}$, is $18 \mathrm{~dB}$ according to (2). The beamwidth of the array pattern is approximately $5^{\circ}$ and $6^{\circ}$ on the Az- and El-planes $(x-z$ and $y-z$ planes), respectively, and $\pm 25^{\circ}$ beams are capable of electronic steering in the coverage region. Furthermore, when the MSE adaptive algorithms in (5) and (6) are applied, the massive MIMO can synthesize the radiation pattern with nulls in the interference directions (i.e., $\left(\theta^{\circ}, \phi^{\circ}\right)=\left(13^{\circ}, 0^{\circ}\right)$, $\left.\left(30^{\circ}, 0^{\circ}\right),\left(50^{\circ}, 0^{\circ}\right)\right)$ to increase the system SNR (Figure 3(b)). Figures 3(c) and 3(d) illustrate plots of the 3D and 2D contour radiation patterns of the array, respectively.

2.2. Microstrip Antenna Array Design. Considering the drawbacks of working at the Ka band frequencies discussed in Section 1, we employed the microstrip antennas for this prototype study because the manufacturing process for microstrip type antenna, with the help of modem-printed circuit technology adapted to testing and constructing purposes, is simpler, less expensive, and faster than that of other types of antennas. Moreover, if manufactured carefully, the products are almost identical, implying that verification of the design microstrip prototype antenna will also be satisfactory for other copies of the antenna. However, the microstrip antennas have notable disadvantages, such as low power handling and narrow frequency bandwidth. 


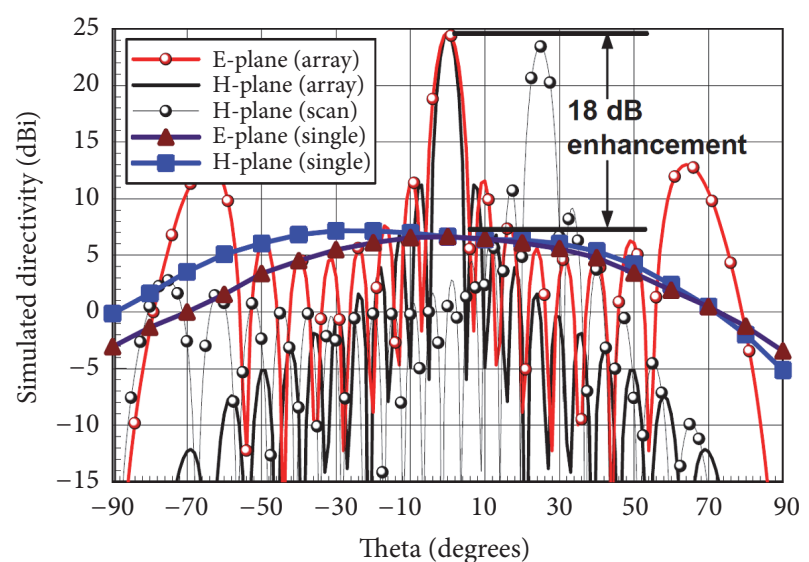

(a)

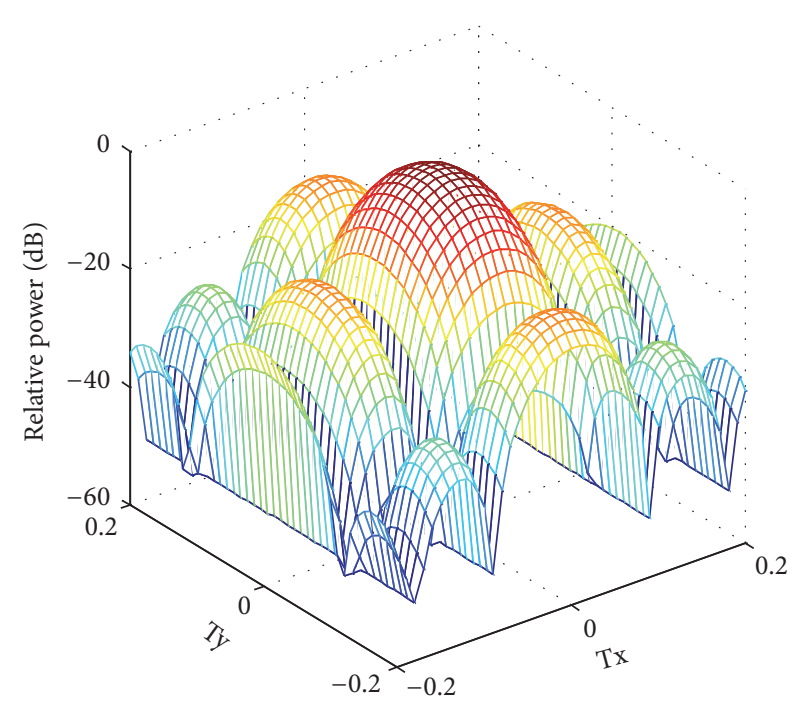

(c)

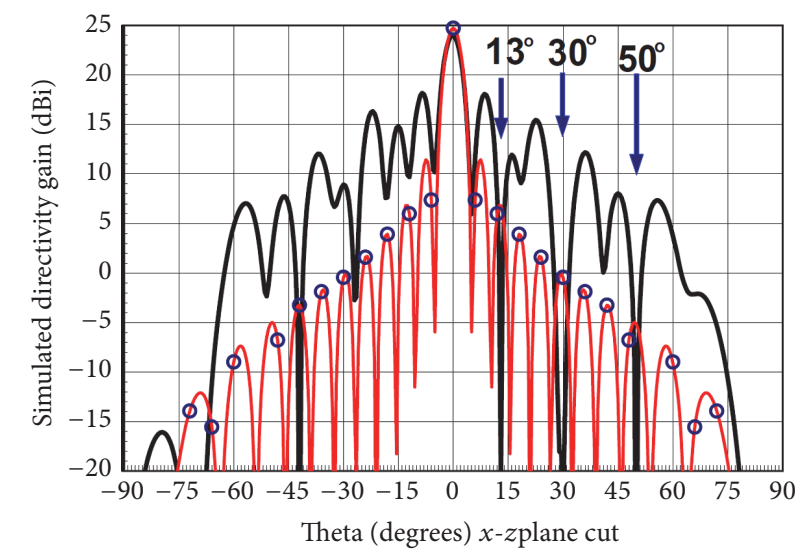

(b)

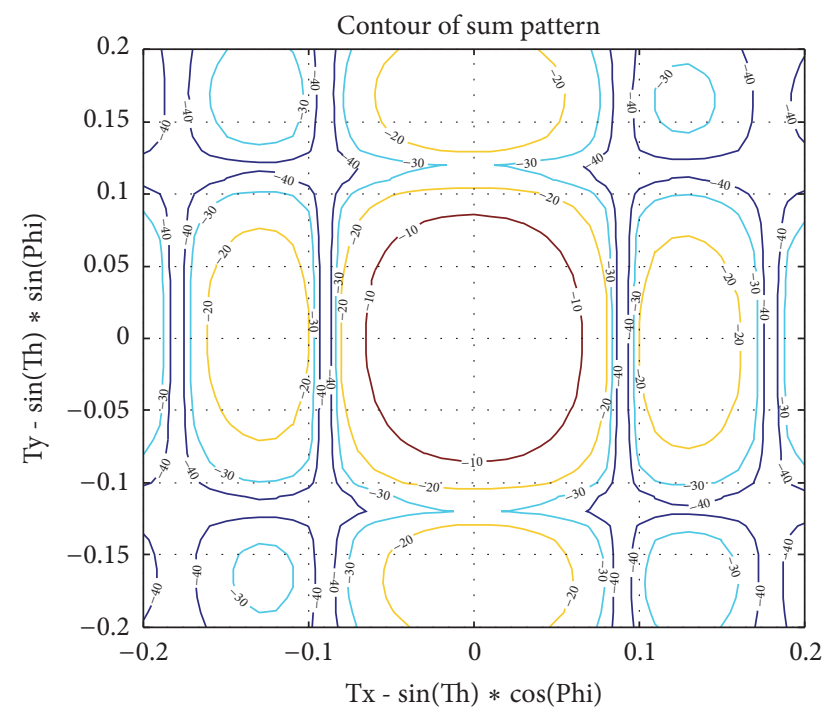

(d)

FIGURE 3: (a) Comparison of the simulated radiation patterns of a single antenna using HFSS and a 64-element array antenna with element spacing of $d x=5.5 \mathrm{~mm}$ and $d y=8 \mathrm{~mm}$ through (2) in E- and H-planes ( $x-z$ and $y-z$ planes). (b) Comparison of the H-plane radiation pattern with and without the adaptive algorithm, demonstrating the nulls on the interference directions $\left(\theta^{\circ}\right.$ and $\left.\phi^{\circ}\right)$ of $13^{\circ}$ and $0^{\circ}, 30^{\circ}$ and $0^{\circ}$, and $50^{\circ}$ and $0^{\circ}$, respectively. (c) $3 \mathrm{D}$ radiation pattern of the array. (d) $2 \mathrm{D}$ contour of the radiation patterns.

Many feeding design methods can be applied to excite microstrip patch antennas, but a perpendicular coaxial transition is the most suitable for $\mathrm{mmW}$ applications, because this method's measurements indicate a return loss better than $14 \mathrm{~dB}$ and an insertion loss better than $0.4 \mathrm{~dB}$ from $\mathrm{DC}$ to $40 \mathrm{GHz}[21,22]$. Therefore, we proposed the $\mathrm{mmW}$ antenna array comprising 64-element perpendicular coaxfed microstrip patch antennas that resonate at $38 \mathrm{GHz}$ (Figure 4). The mmW microstrip patch array is integrated with eight subarrays, each comprising eight microstrip patches printed on the RF substrate with a thickness of 15 mil. The element spacing of the array is arranged in a triangular lattice $(d x=5.5 \mathrm{~mm} ; d y=8.5 \mathrm{~mm})$ to obtain a beam scanning azimuth angle of $\pm 25^{\circ}$ when maintaining maximum room to allocate the modified $\mathrm{k}$-connector.

A key parasite of perpendicular transition is the inductance triggered by ground currents that must flow around the circumference of the coax outer conductor to reach the underside of the microstrip section. Therefore, in each subarray, substrates with the printed microstrip patches were soldered on a modified $\mathrm{k}$-connector with four ground pins on the four corners of the connector base to secure the antenna (Figure 5(a)). After all of the subarrays were integrated, nine screws were used to fix the RF substrate of each subarray onto the back plane of the array (Figure 5(b)). Subsequently, the array was placed in a tin stove for wave soldering reflow. After all subarrays were soldered on the back plane of the array, all of the screws were removed.

\section{Numerical and Experimental Study}

The design approach of the microstrip patch printed on the LTCC substrate with a dielectric constant of 5.9 and thickness of 15 mil was verified using HFSS (Figure 6). The transition effects of the coaxially fed launcher were considered in the simulation. 


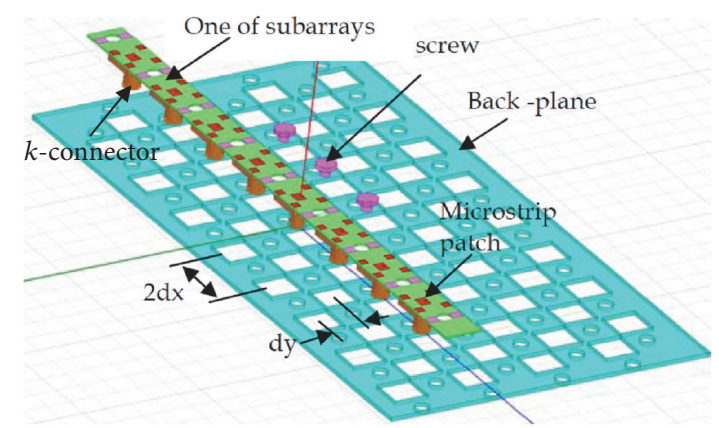

FIGURE 4: Design concept of the proposed mmW microstrip antenna array.

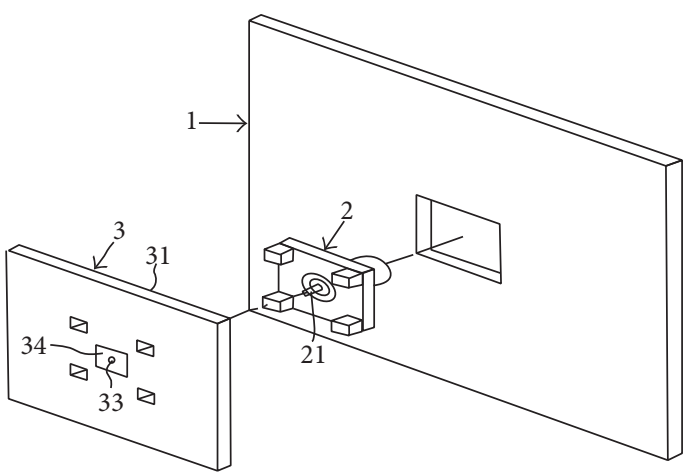

(a)

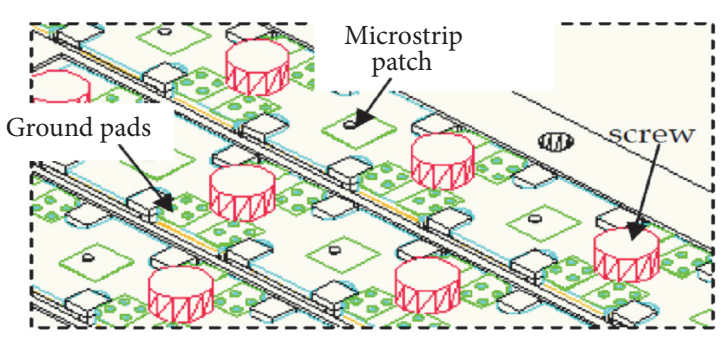

(b)

Figure 5: (a) Design of a single microstrip patch. (b) Close look of the assembled array.

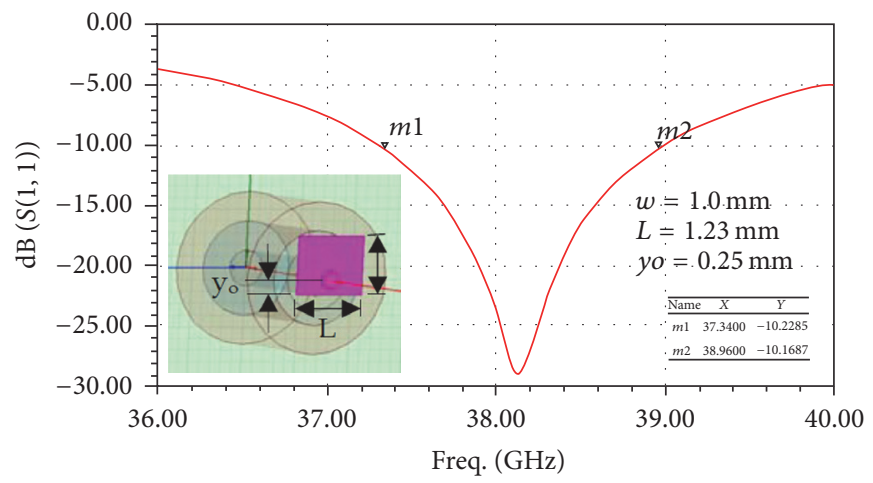

FIGURE 6: Simulated $S_{11}$ of a single microstrip antenna patch; inset shows the simulation layout.

The simulation results (Figure 6) showed a return loss of less than $10 \mathrm{~dB}$ at the frequencies of interest ranging from 37.34 to $38.89 \mathrm{GHz}$. To experimentally verify this design approach, two identical $\mathrm{mmW}$ microstrip patch antenna arrays were built for testing (Figure 7). The measurement setup was established by connecting one patch with two $\mathrm{mmW}$ microstrip patch arrays, which were placed face-toface $19.5 \mathrm{~cm}$ apart (Figure 8 ).
Two-port $S$-parameters were measured using the Rohde \& Schwarz ZVA40 vector network analyzer (Figure 9). The simulated $S_{11}$ (solid line in Figure 9) was computed using a single microstrip patch to reduce computing time but the measured $S_{11}$ (the solid line with diamond shapes) and $S_{22}$ (the solid line with rectangular shapes) corresponded to the center element of two microstrip antenna arrays (Figure 8). Thus, the discrepancy between the measured and simulated 


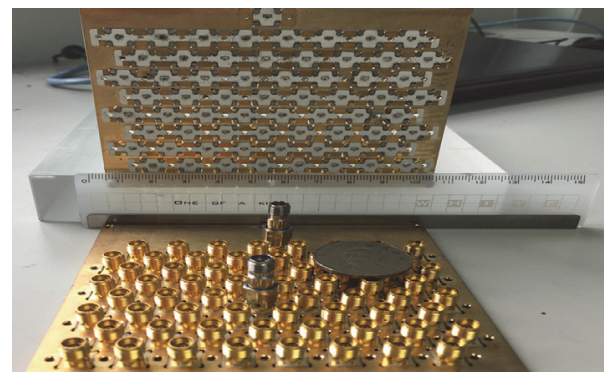

FIGURE 7: Photograph of two identical mmW microstrip arrays for testing.

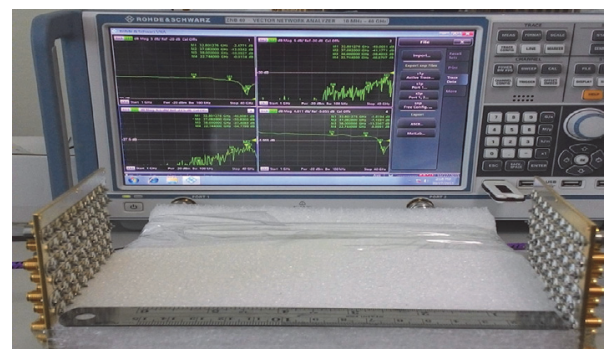

FIGURE 8: Measurement setup established by connecting one patch to two arrays, placing two arrays face-to-face at a distance of $R=19.5 \mathrm{~cm}$, and testing the 2-port S-parameters using the Rohde \& Schwarz ZVA40 vector network analyzer.

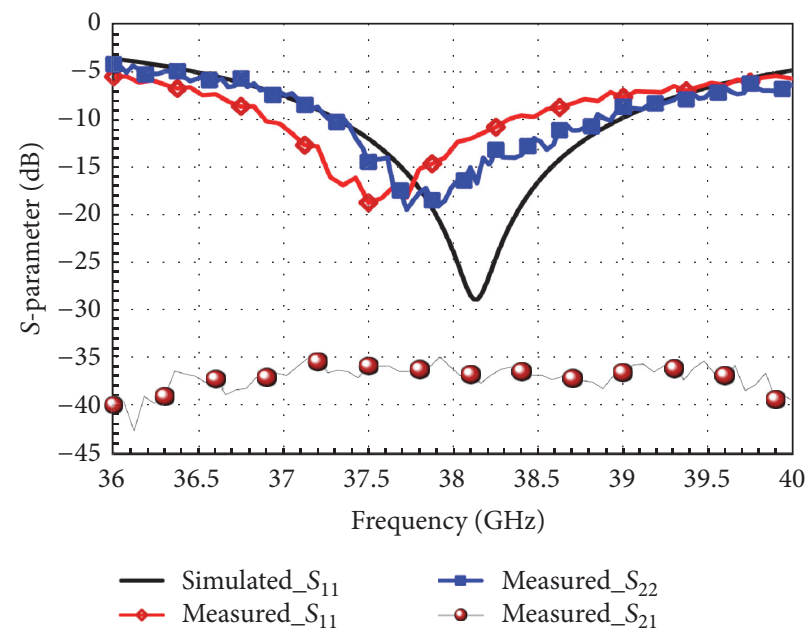

FIGURE 9: Measured S-parameters of the test setup (Figure 8).

data (Figure 9) can be attributed to the reactive loading effect caused by the mutual coupling.

Next, the Friis power transmission formula [23] was used to calculate the maximum antenna power gain (in the central forward direction of the antenna):

$$
\mathbf{G}_{\mathbf{r}} \mathbf{G}_{\mathbf{t}}=\mathbf{G}^{2}=\left(\frac{\mathbf{P}_{\mathbf{r}}}{\mathbf{P}_{\mathbf{t}}}\right)\left(\frac{4 \pi \mathbf{R}}{\lambda_{0}}\right)^{2}=\left|\mathbf{S}_{21}\right|^{2}\left(\frac{4 \pi \mathbf{R}}{\lambda_{0}}\right)^{2},
$$

where $\mathbf{G}_{\mathbf{r}}$ and $\mathbf{G}_{\mathbf{t}}$ are the power gains of the receiving and transmitting antennas, respectively, and $\mathbf{P}_{\mathbf{t}}$ and $\mathbf{P}_{\mathbf{r}}$ are the transmitted and received powers, respectively. Because the two antennas are identical, $\mathbf{G}_{\mathbf{t}}=\mathbf{G}_{\mathbf{r}}=\mathbf{G}$, and the power ratio $\mathbf{P}_{\mathbf{r}} / \mathbf{P}_{\mathbf{t}}$ is the measured direct transmission coefficient $\left|\mathbf{S}_{21}\right|^{2}$, which was obtained using the vector network analyzer. By substituting the measured $\left|\mathbf{S}_{21}\right|$ into (9), the measured maximum antenna power gain was plotted against frequencies ranging from 36.0 to $40 \mathrm{GHz}$ to compare with simulation data (Figure 10); they indicated an acceptable agreement. The measured gain of a single microstrip patch was approximately $6.8 \mathrm{dBi}$ at $38 \mathrm{GHz}$.

Finally, the radiation pattern at the center element of the prototype $\mathrm{mmW}$ array (Figure 7) was measured at OIT 


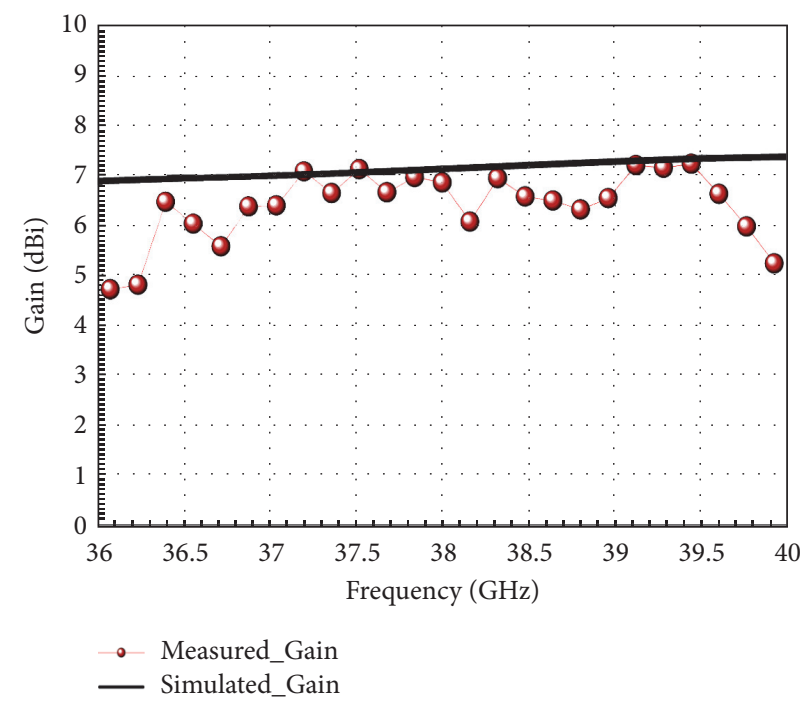

FIGURE 10: Comparison of the gain measured by (9) and the gain simulated using HFSS on a single microstrip patch antenna.

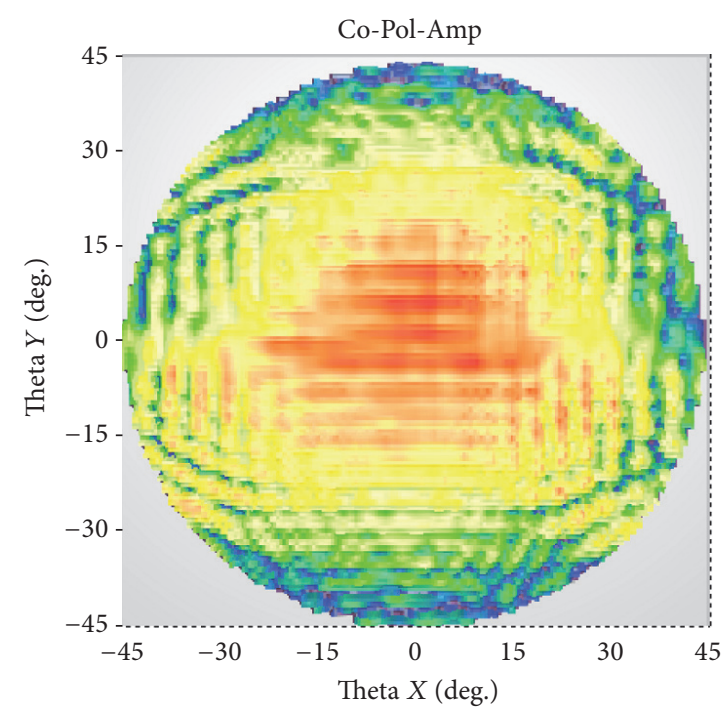

(a) Amplitude

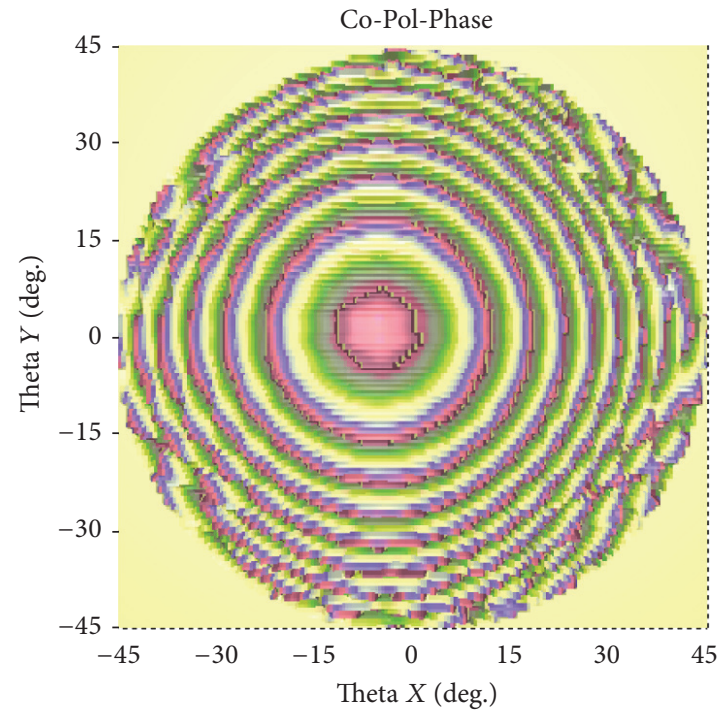

(b) Phase

FIgURE 11: Measured (a) amplitude and (b) phase of the aperture field of the mmW microstrip antenna array excited at the center element.

near- and far-field test ranges. The measured amplitude and phase of the aperture field are plotted in Figures 11(a) and 11(b), respectively. Meanwhile, Figures 12(a) and 12(b) display photographs of the tested setup, and Figure 12(c) illustrates plots of the measured Co-Pol and Cross-Pol for the 2D radiation pattern cuts in the $x-y$ and $x-z$ planes at $37.75 \mathrm{GHz}$.

\section{Conclusion}

Our design prototype for an $\mathrm{mmW}$ microstrip antenna array operating at the $\mathrm{Ka}$ band frequency range for $5 \mathrm{G}$ communication system applications is presented using 64element microstrip patches printed on an LTCC substrate with a thickness of $15 \mathrm{mil}$ and a dielectric constant of 5.9.
Our numerical and experimental study revealed acceptable agreement between the measured and simulated return losses and antenna power gain performance. This confirms that a microstrip patch antenna array with a high radiation gain performance in an $\mathrm{mmW}$ range is feasible for future $5 \mathrm{G}$ applications.

\section{Competing Interests}

The authors declare that they have no competing interests.

\section{Acknowledgments}

The authors thank the Chung-Shan Institute of Science and Technology (Taiwan) for financially supporting this research 


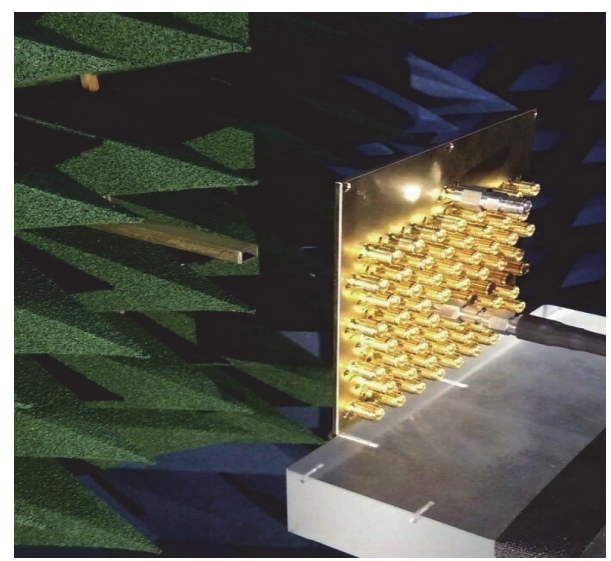

(a)

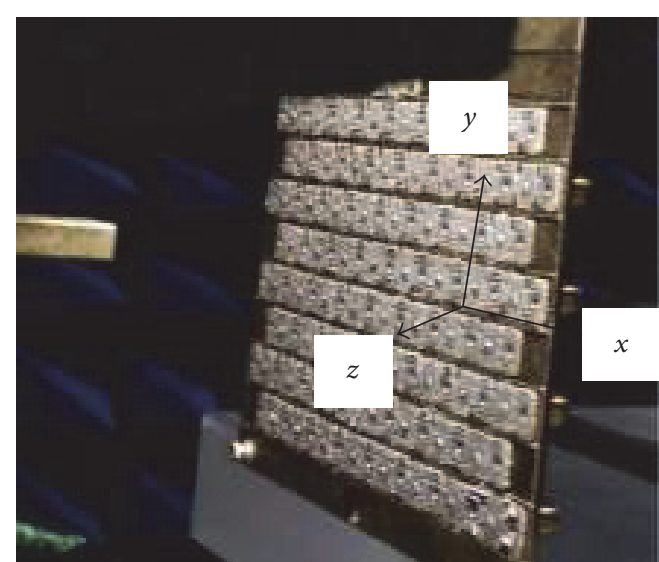

(b)

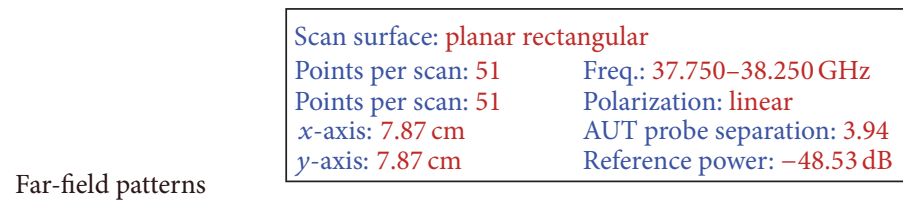
Frequency: 37.7500

Co-Pol and X-Pol-Amp

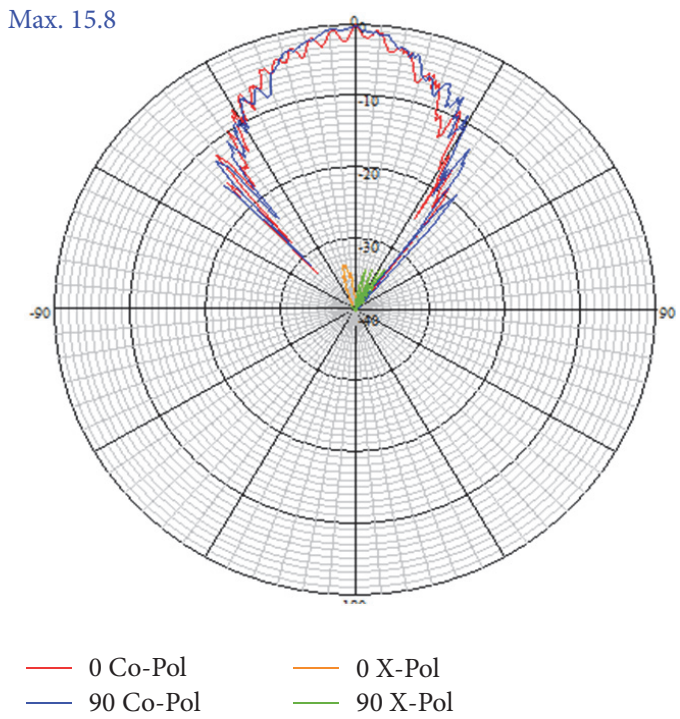

(c)

FIGURE 12: (a) Amplitude and (b) phase photographs of the tested setup of the mmW microstrip antenna array. (c) Measured 2D Co-Pol and Cross-Pol radiation patterns.

(CSIST-A7I-V1XX) and thank Kevin Peng for assisting in the manufacture and integration of the array.

\section{References}

[1] J. Thompson, X. Ge, H.-C. Wu et al., "5G wireless communication systems: prospects and challenges [Guest Editorial]," IEEE Communications Magazine, vol. 52, no. 2, pp. 62-64, 2014.

[2] M. S. Pandey, M. Kumar, A. Panwar, and I. Singh, "A survey: wireless mobile technology generations with 5G," International Journal of Engineering Research and Technology, vol. 2, no. 4, 2013.
[3] Ericsson, "More than 50 billion connected devices," White Paper. [Online], http://www.akos-rs.si/files/Telekomunikacije/ Digitalna_agenda/Internetni_protokol_Ipv6/More-than-50-billion-connected-devices.pdf.

[4] Cisco, Cisco Visual Network Index: Global Mobile Traffic Forecast Update, 2013.

[5] Ericsson, "Traffic and market data report," 2011.

[6] UMTS Forum, Mobile Traffic Forecasts: 2010-2020 Report, UMTS Forum, Zürich, Switzerland, 2011.

[7] P. E. Mogensen, K. Pajukoski, B. Raaf et al., "B4G local area: high level requirements and system design," in Proceedings of the 
IEEE Globecom Workshops (GC Wkshps '12), pp. 613-617, IEEE, Anaheim, Calif, USA, December 2012.

[8] T. S. Rappaport, J. N. Murdock, and F. Gutierrez, "State of the art in $60-\mathrm{GHz}$ integrated circuits and systems for wireless communications," Proceedings of the IEEE, vol. 99, no. 8, pp. 1390-1436, 2011.

[9] F. Khan and Z. Pi, "Millimeter wave mobile broadband (MMB): unleashing the 3-300 GHz spectrum," in Proceedings of the 34th IEEE Sarnoff Symposium, March 2011.

[10] Z. Pi and F. Khan, "An introduction to millimeter-wave mobile broadband systems," IEEE Communications Magazine, vol. 49, no. 6, pp. 101-107, 2011.

[11] P. Pietraski, D. Britz, A. Roy, R. Pragada, and G. Charlton, "Millimeter wave and terahertz communications: feasibility and challenges," ZTE Communications, vol. 10, no. 4, pp. 3-12, 2012.

[12] Y. P. Zhang and D. Liu, "Antenna-on-chip and antenna-inpackage solutions to highly integrated millimeter-wave devices for wireless communications," IEEE Transactions on Antennas and Propagation, vol. 57, no. 10, pp. 2830-2841, 2009.

[13] S. Rangan, T. S. Rappaport, and E. Erkip, "Millimeter-wave cellular wireless networks: potentials and challenges," Proceedings of the IEEE, vol. 102, no. 3, pp. 366-385, 2014.

[14] B. Widrow, L. J. Griffiths, P. E. Mantey, and B. B. Goode, "Adaptive antenna systems," Proceedings of the IEEE, vol. 55, no. 12, pp. 2143-2159, 1967.

[15] W. F. Gabriel, "Adaptive array-an introduction," Proceeding of IEEE, vol. 55, no. 12, pp. 2144-2159, 1967.

[16] C.-N. Hu, "A novel method for calibrating deployed active antenna arrays," IEEE Transactions on Antennas and Propagation, vol. 63, no. 4, pp. 1650-1657, 2015.

[17] J. E. Hudson, Adaptive Array Principles (IEEE Electromagnetic Waves Series), Institution of Engineering and Technology, Stevenage, UK, 1981.

[18] J. G. Proakis, Digital Communications, McGraw-Hill, New York, NY, USA, 4th edition, 2001.

[19] M. Wennström, On MIMO systems and adaptive arrays for wireless communication [Ph.D. thesis], Uppsala University, Uppsala, Sweden, 2002.

[20] G. Tsoulos, M. Beach, and J. McGeehan, "Space division multiple access (SDMA) field trials. Part 2: calibration and linearity issues,", IEE Proceedings-Radar, Sonar and Navigation, vol. 145, no. 1, pp. 79-84, 1998.

[21] M. Morgan and S. Weinreb, "A millimeter-wave perpendicular coax-to-microstrip transition," in Proceedings of the IEEE MSSS International Microwave Symposium Digest, pp. 817-820, June 2002.

[22] J. Chenkin, "DC to $40 \mathrm{GHz}$ coaxial-to-microstrip transition for 100-_m-thick GaAs substrates," IEEE Transactions on Microwave Theory and Techniques, vol. 37, pp. 1147-1150, 1989.

[23] D. K. Misra and K. M. Devendra, Radio-Frequency and Microwave Communications Circuits-Analysis and Design, Ch. 2.4 Antenna Systems, John Wiley \& Son, Inc, Hoboken, NJ, USA, 2nd edition, 2004. 


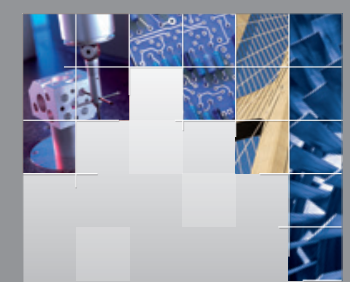

\section{Enfincering}
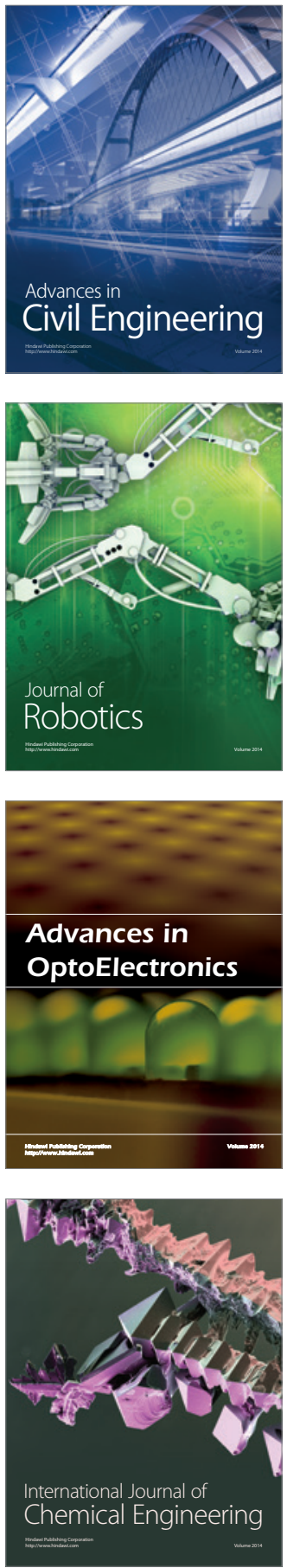

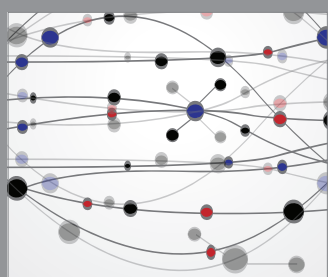

The Scientific World Journal

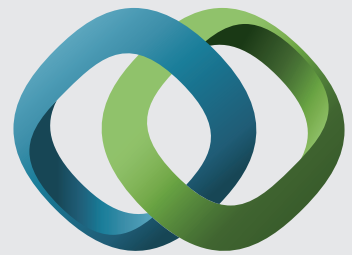

\section{Hindawi}

Submit your manuscripts at

http://www.hindawi.com
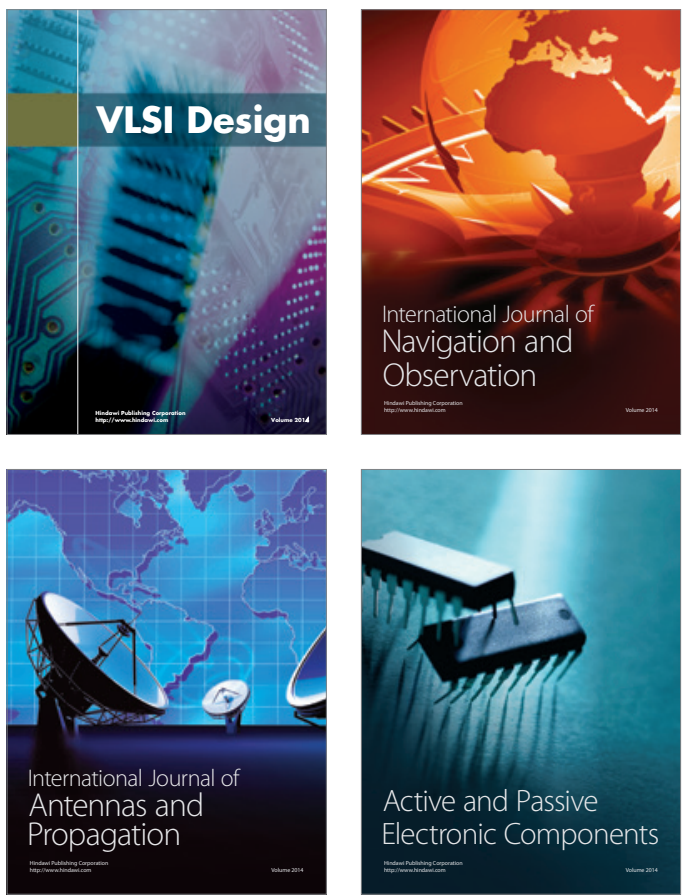
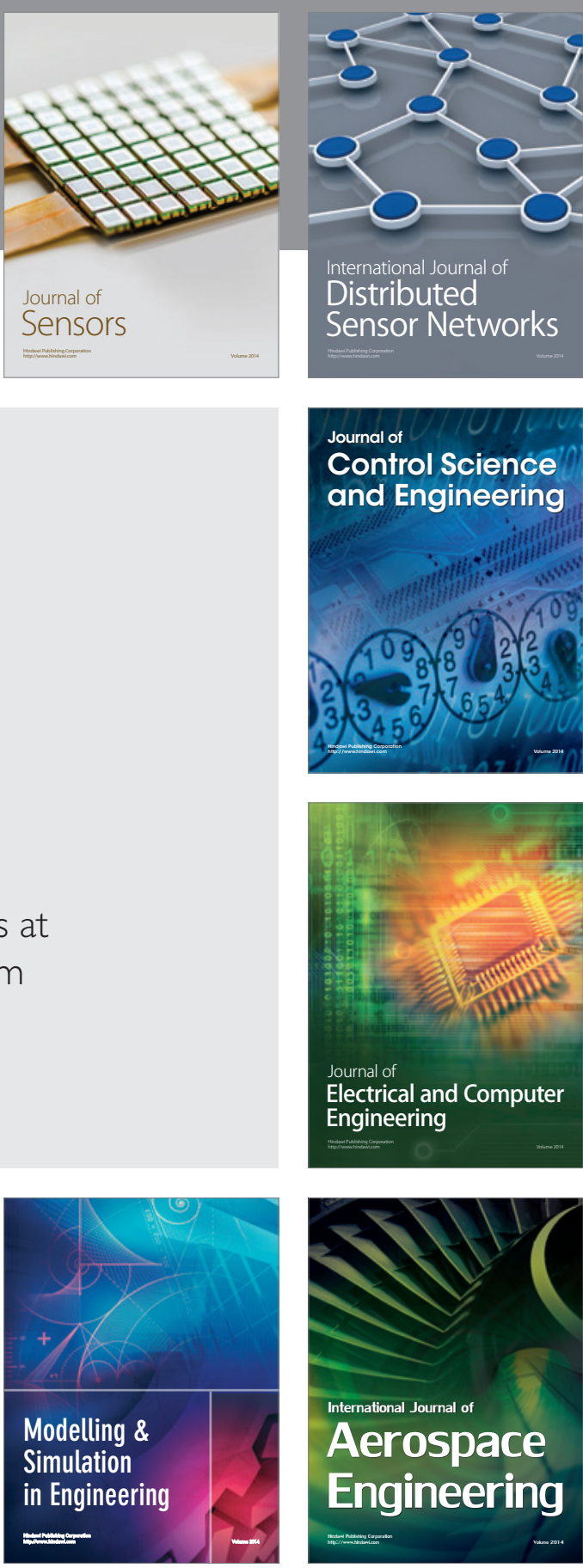

International Journal of

Distributed

Sensor Networks

Journal of

Control Science

and Engineering
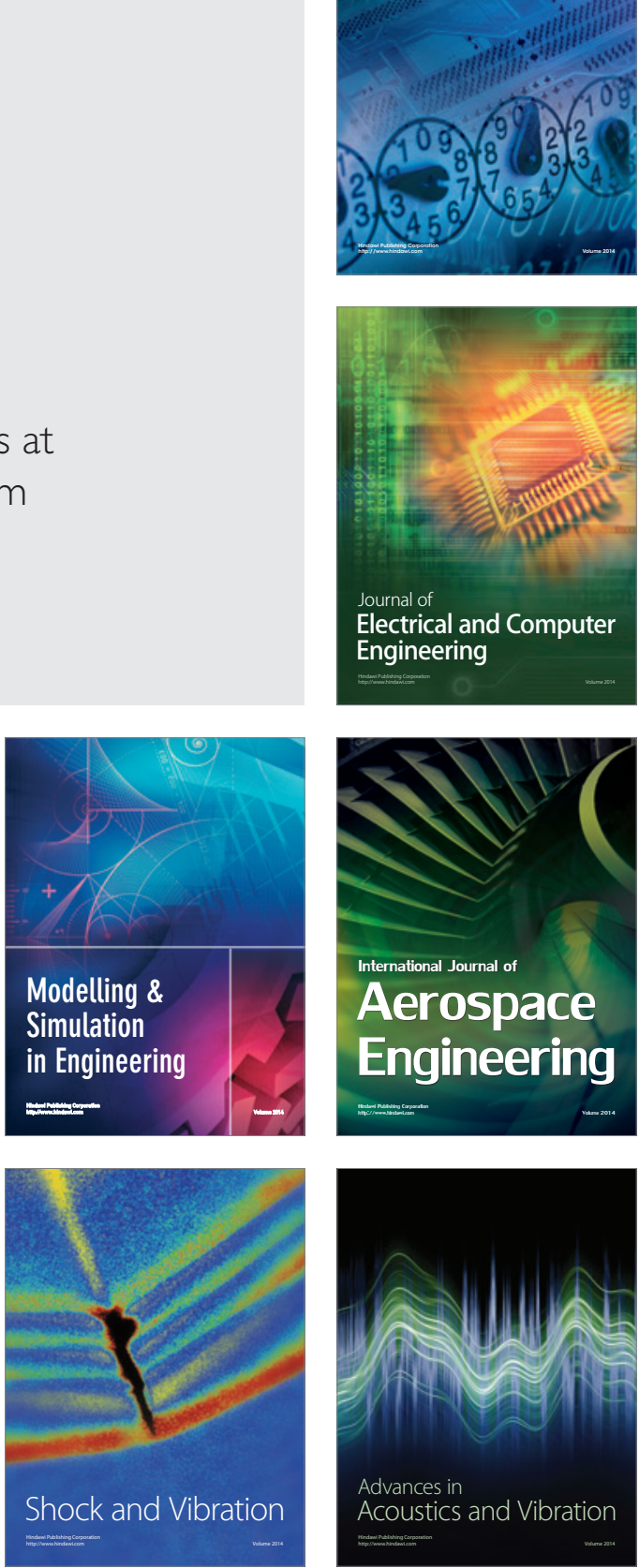